\title{
Nanopillars array for surface enhanced Raman scattering
}

A. S.P. Chang, M. Bora, H. T. Nguyen, E. M. Behymer, C. C. Larson, J. A. Britten, J. C. Carter, T. C. Bond

April 20, 2011

SPIE Defense, Security \& Sensing Orlando, FL, United States April 25, 2011 through April 29, 2011 
This document was prepared as an account of work sponsored by an agency of the United States government. Neither the United States government nor Lawrence Livermore National Security, LLC, nor any of their employees makes any warranty, expressed or implied, or assumes any legal liability or responsibility for the accuracy, completeness, or usefulness of any information, apparatus, product, or process disclosed, or represents that its use would not infringe privately owned rights. Reference herein to any specific commercial product, process, or service by trade name, trademark, manufacturer, or otherwise does not necessarily constitute or imply its endorsement, recommendation, or favoring by the United States government or Lawrence Livermore National Security, LLC. The views and opinions of authors expressed herein do not necessarily state or reflect those of the United States government or Lawrence Livermore National Security, LLC, and shall not be used for advertising or product endorsement purposes. 


\title{
Nanopillars array for surface enhanced Raman scattering
}

\author{
Allan S. P. Chang*a , Mihail Bora ${ }^{a}$, Hoang T. Nguyen ${ }^{a}$, Elaine M. Behymer ${ }^{a}$, Cindy C. Larson ${ }^{a}$, \\ Jerald A. Britten ${ }^{\mathrm{a}}$, J. Chance Carter ${ }^{\mathrm{a}}$, and Tiziana C. Bond*a \\ ${ }^{a}$ Lawrence Livermore National Laboratory, 7000 East Ave., Livermore, CA, USA 94550
}

\begin{abstract}
We present a new class of surface-enhanced Raman scattering (SERS) substrates based on lithographically-defined twodimensional rectangular array of nanopillars. Two types of nanopillars within this class are discussed: vertical pillars and tapered pillars. For the vertical pillars, the gap between each pair of nanopillars is small enough $(<50 \mathrm{~nm})$ such that highly confined plasmonic cavity resonances are supported between the pillars when light is incident upon them, and the anti-nodes of these resonances act as three-dimensional hotspots for SERS. For the tapered pillars, SERS enhancement arises from the nanofocusing effect due to the sharp tip on top. SERS experiments were carried out on these substrates using various concentrations of 1,2 bis-(4-pyridyl)-ethylene (BPE), benzenethiol (BT) monolayer and toluene vapor. The results show that SERS enhancement factor of over $0.5 \times 10^{9}$ can be achieved, and BPE can be detected down to femtomolar concentration level. The results also show promising potential for the use of these substrates in environmental monitoring of gases and vapors such as volatile organic compounds.
\end{abstract}

Keywords: Surface Enhanced Raman Scattering, spectroscopy, sensing, nanostructures

\section{INTRODUCTION}

Surface-Enhanced Raman Scattering (SERS) is a powerful analytical technique due to its specificity and high sensitivity up to single-molecule level $[1,2]$. There is also increasing interest in using SERS for gas and vapor detection and sensing [3-6] due to its potential for remote and in situ analysis, in addition to its high sensitivity and molecule-specific fingerprinting. The substrate on which the SERS is performed is often the critical component for successful detection and identification of the targeted molecules. The common ways to produce SERS-active substrates such as thin metal island films [7], electrochemical roughening [8], metallic nanoparticles [9], and self-assembly [10] often results in structures that are random in nature and/or are limited in control of their structural geometry. This can lead to issues with uniformity, reproducibility and repeatability that hinder the large-scale use of SERS. It is also difficult to optimize the geometry of these substrates to attain the maximum possible enhancement. A solution to these challenges is through the use of substrates that are precisely defined regular nanostructures obtained by lithography [11,12]. Furthermore, a lithographically-defined SERS substrate can offer more degree of freedom in the design and tuning of its geometrical parameters, thereby allowing optimization that can lead to higher SERS enhancement factor.

We present a new class of SERS substrates based on lithographically-defined two-dimensional rectangular array of nanopillars. Two types of nanopillars within this class are discussed: vertical nanopillars (VNPs) and tapered nanopillars (TNPs). As these substrates are created by well-established, high-precision planar nanofabrication techniques and processes, their geometrical parameters can be precisely controlled and fine-tuned, thereby allowing optimization of their performance. Another advantage of lithographically-defined SERS substrates is that they can be fabricated with high uniformity and reproducibility over large area, and are well-suited for mass-production. In addition, these nanopillars are closely-spaced (pitch $=350 \mathrm{~nm}$ here) and thus allow for a very high density of SERS 'hotspots' that can be pre-defined. In principle, the hotspot density is only limited by the resolution of nanolithography, which is itself constantly improving due to demands of the semiconductor industry.

The VNP type of SERS substrate is comprised of arrays of closely-spaced straight nanopillars. The gap between each pair of nanopillars is small enough $(<50 \mathrm{~nm})$ such that highly confined plasmonic cavity resonances are supported between the pillars when light is incident upon them, and the anti-nodes of these resonances act as three-dimensional

*chang43@1lnl.gov, phone1925 423-1856; bond7@1lnl.gov, phone1925 423-2205 
hotspots for SERS [13]. An interesting result arising from this cavity effect is that the resonance of these nanostructures can be tuned by varying the depth of the pillars, which in effect varies the cavity length. As these nanostructures are precisely defined by lithography, they offer the unique capability of being tailored for the specific applications. As a demonstration of their potential use for SERS, SERS experiments were carried out on these substrates using various concentrations of 1,2 bis-(4-pyridyl)-ethylene (BPE) dissolved in methanol. The results show that this type of substrate can possess spatially-averaged SERS enhancement factor of over $0.5 \times 10^{9}$, and BPE can be detected at femto-molar concentration level by these substrates.

On the other hand, the TNP substrates are designed to have tapered pillars with sharp tip on top (ideally, cones) and are over-coated with metal (e.g. Ag) deposited at an angle. They have previously shown SERS enhancement factor of around $10^{8}[14]$ due to the nanofocusing effect. SERS experiments were carried out on these substrates using toluene vapor as the analyte. There is important need for compact, field-deployable, sensitive, and molecule-specific detection techniques for volatile organic compounds (VOCs) such as toluene due to their prevalence as air-pollutants as well as importance in national security applications. The VOCs have found widespread use in industry, particularly as solvents; and yet they possess significant health risks to humans and there is thus increasing interest in their rapid detection. Our results show that saturated toluene vapor can be detected effectively at room temperature by the TNP substrate and there is promising potential for the use of these substrates in detecting VOCs.

\section{EXPERIMENTS}

\subsection{Fabrication of nanopillars SERS substrates}

The nanopillars substrate was fabricated by holographic interference lithography [15] over 4-inch fused silica wafers that are coated with half-micron thick photoresist. The laser wavelength for the lithography is $413 \mathrm{~nm}$ and the dose is around $40 \mathrm{~mJ} / \mathrm{cm}^{2}$. The resultant photoresist structure is a two-dimensional periodic array of pillars with pitch of $350 \mathrm{~nm}$ and diameter of $150 \mathrm{~nm}$. The exposed wafers are cleaved into smaller pieces for subsequent processing. The patterned photoresist acts as the etching mask in a subsequent step of ion beam milling.

To obtain the straight pillars, the silicon dioxide is etched $500 \mathrm{~nm}$ and the residual photoresist mask is washed away. A gold $(\mathrm{Au})$ metal layer of $80 \mathrm{~nm}$ is then deposited conformingly over the patterned straight glass pillars using atomic layer deposition (ALD). Since the deposition is highly conformal in ALD, the gap between individual pillars closes up during the deposition. Furthermore, the deposition thickness is highly controllable. This effect is used to precisely control the width of the gap between pillars by controlling the depth of metal deposition. Using this process, straight gold-coated pillar arrays with pillar-gap width of less than $50 \mathrm{~nm}$ can be achieved reliably (fig. 1a-b).

To obtain the tapered pillars, the silicon dioxide is over-etched in the ion beam milling process. As there is side-way etching, an undercut will start to develop under the photoresist. Through careful testing and calibration, the desired tapered pillar shape with sharp tips on top can be achieved. Pillar height can be varied between $250 \mathrm{~nm}$ to $1000 \mathrm{~nm}$. A 40 nm layer of silver $(\mathrm{Ag})$ was then e-beam evaporated onto it at an angle of $30^{\circ}$ to make it SERS-active (fig. 1c-d).

\subsection{Preparation of BPE solutions}

Solid powders of BPE (purity > $99.9 \%$ ) were obtained commercially through Sigma-Aldrich. $17 \mathrm{mg}$ of BPE powder is carefully weighted on an electronic weight scale and dissolved in $7 \mathrm{ml}$ of methanol at room temperature. The solution was put in ultrasonic bath for 3 minutes. This results in a 13mM BPE solution. $1 \mathrm{ml}$ of this solution is then pipetted out and mixed with $25 \mathrm{ml}$ of methanol to obtain a more dilute solution. By successively repeating this step, a low concentration down to $55 \mathrm{fM}$ was obtained.

\subsection{Bubbling of Toluene}

Nitrogen carrier gas was fed using Teflon tubings and bubbled into toluene liquid (Sigma-Aldrich, purity $>99.9 \%$ ) held in a glass bubbler at a temperature of $20{ }^{\circ} \mathrm{C}$. The flow is controlled by a regulator set at 2 psi. The toluene vapor is assumed to saturate the resultant gas mixture [16]. This gas mixture was then fed into a gas cell (Linkam THMS600) with our SERS substrate placed on top of a temperature-controlled stage inside the gas cell. The gas flow is continuous and exits from the gas cell into an exhaust. 


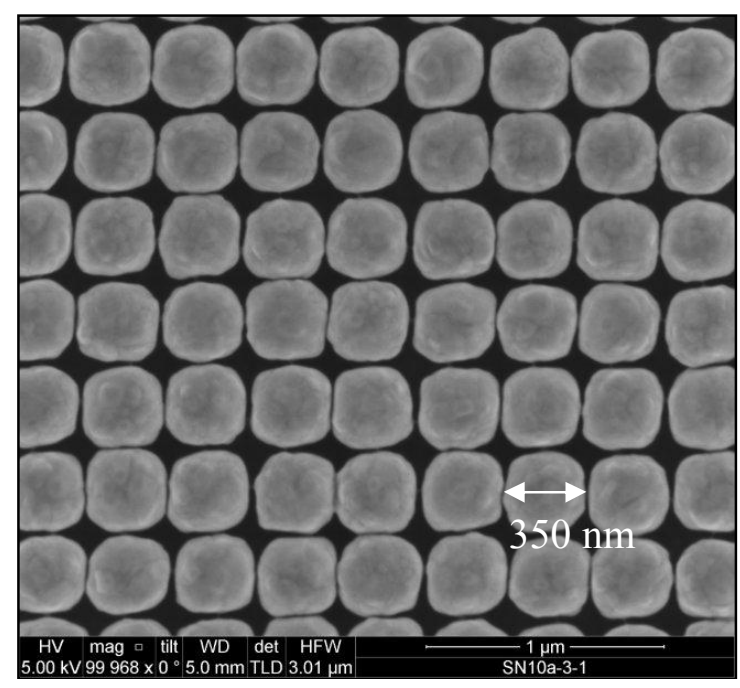

a.)

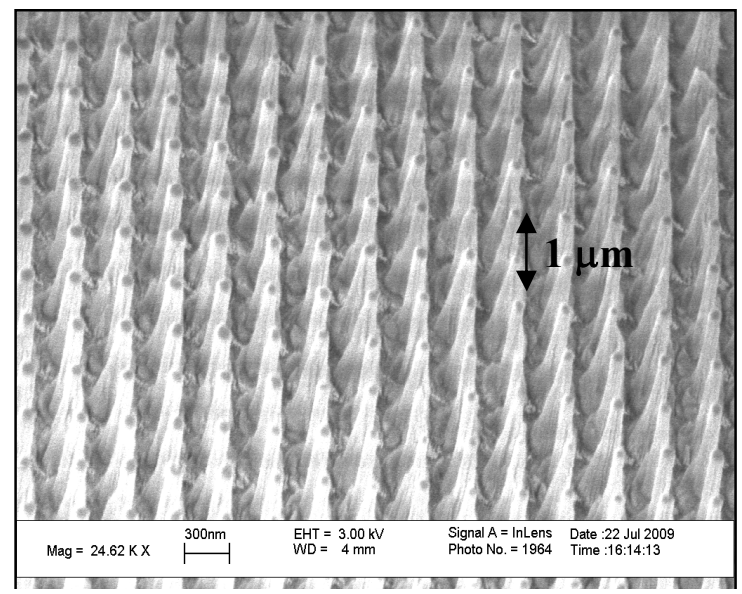

c.)

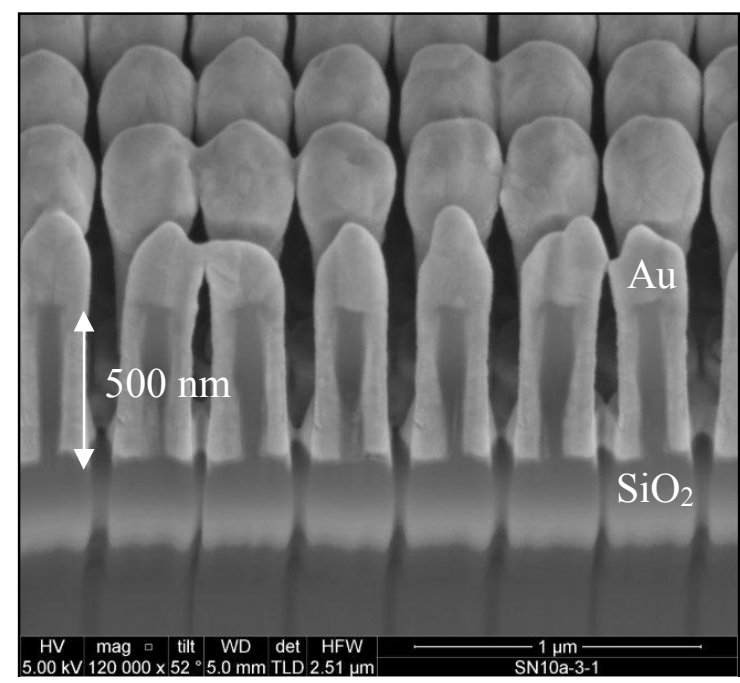

b.)

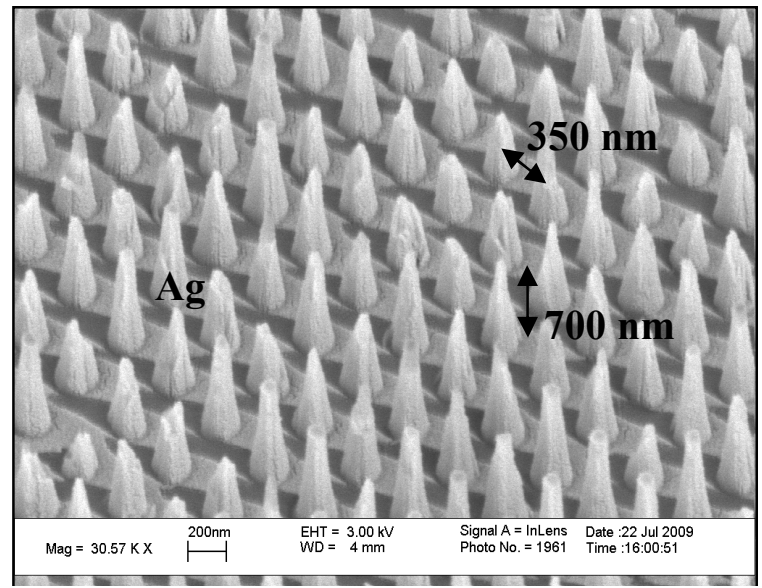

d.)

Fig. 1. SEM images of nanopillars arrays for SERS applications. a.) Top view of Au-coated vertical nanopillars (VNPs); b.) Tilted view of Au-coated VNPs; c.) Tilted view of Ag-coated tapered nanopillars (TNPs) with $1000 \mathrm{~nm}$ depth; d.) Tilted view of Ag-coated TNPs with $700 \mathrm{~nm}$ depth.

\subsection{Spectroscopy}

To obtain SERS spectra of BPE, a commercially available petri dish with a clear vial at bottom is used. Solution of BPE with volume of $0.5 \mathrm{ml}$ was pipetted to fill this vial. VNP sample is then immersed in the solution, facing down. The spectroscopy was done using a diode-pumped solid state laser (Crystal Laser RCL-040-660-S) at a wavelength of 660 $\mathrm{nm}$ with power of $14 \mathrm{~mW}$ measured with a calibrated power meter. The light is focused using a 20x objective lens (Nikon, NA $=0.4, \mathrm{wd}=11 \mathrm{~mm}$ ) onto the sample surface in an inverted microscope setup. The SERS signal is collected using the sample lens and is fed into a grating based spectrometer (Acton Research Corp, SpectraPro-300i), which spectrally dispersed the signal onto a liquid nitrogen-cooled $\left(-120^{\circ} \mathrm{C}\right)$ CCD camera (Roper Scientific, Model 73460001). Data collection was done through the WinSpec software. 
For the toluene vapor measurement using the TNPs, a diode-pumped solid state laser (OEM Lasers) emitting at $532 \mathrm{~nm}$ was used. A Kaiser Optics fiber probe (MK II) delivers the laser light through a window on the gas cell, and the Raman signal was collected by the same fiber probe. The delivered laser power after the fiber probe is measured to be $4 \mathrm{~mW}$. Focusing is achieved by vertically moving the gas cell, which is fixed on a precision x-y-z translational stage. The SERS signal is fed into a grating based spectrometer (Kaiser Optics), which spectrally dispersed the signal onto a TE-coolercooled $\left(-70^{\circ} \mathrm{C}\right) \mathrm{CCD}$ camera (Princeton Instruments). Data collection was done through the WinSpec software.

\section{RESULTS AND DISCUSSION}

\subsection{SERS of BPE using Au-coated vertical nanopillar (VNP) substrate}

The SERS spectra of BPE at concentrations of $35 \mathrm{pM}, 1.4 \mathrm{pM}$, and $55 \mathrm{fM}$ obtained using the VNP substrate depicted in Fig 1 a.)-b.) are shown in fig. 2a. The laser wavelength is $660 \mathrm{~nm}$ and the laser power is $14 \mathrm{~mW}$. The data acquisition time is 10 secs. The plot shows that the characteristic SERS peaks of BPE at $1200(\mathrm{C}=\mathrm{C}$ stretching), 1604 (aromatic ring stretching), and 1635 (in-plane ring mode) are discernible down to concentration of $55 \mathrm{fM}$. The peaks are consistent with what is reported in the literature [17]. Also shown in the plot is background obtained using a flat Au film deposited with the same thickness as the Au coating on the pillars substrates, showing that without the pillars, no discernible BPE peaks are present even at $35 \mathrm{pM}$.

The bulk Raman spectrum of $10 \mathrm{mM}$ of BPE is shown in fig. 2b. This spectrum is obtained using $14 \mathrm{~mW}$ of CW laser power and 300 secs of data acqusition time.

With the data on SERS and Raman measurements in fig. 2, the spatially-averaged SERS enhancement factor can be estimated using the following expression [18]:

$$
E F=\frac{I_{S E R S}}{I_{R S}} \frac{P_{R S} T_{R S}}{P_{S E R S} T_{S E R S}} \frac{N_{R S} L}{N_{S E R S}}
$$

where $I_{S E R S}$ is the SERS intensity, $I_{R S}$ is the Raman intensity, $P_{R S}$ is the laser power for Raman measurement, $P_{S E R S}$ is the laser power for SERS measurement, $T_{R S}$ is the data acquisition time for Raman measurement, $T_{S E R S}$ is the data acquisition time for SERS measurement, $N_{R S}$ is the molecular density of BPE for Raman measurement, $N_{S E R S}$ is the areal density of BPE on the nanopillars substrate, and $L$ is the Raman scattering length.

Taking the $1200 \mathrm{~cm}^{-1}$ SERS band at BPE concentration of $35 \mathrm{pM}$ for calculating $E F$, the SERS intensity underneath this band is calculated to be $I_{S E R S}=4752$, the Raman intensity underneath the $1200 \mathrm{~cm}^{-1}$ band is calculated to be $I_{R S}=1400$. The laser power is the same for both measurements $P_{R S}=P_{S E R S}=14 \mathrm{~mW}$. We have $T_{R S}=300$ secs, and $T_{S E R S}=10$ secs. The Raman spectrum is taken at a BPE concentration of $10 \mathrm{mM}$, thus $N_{R S}=6 \times 10^{18}$ molecules $/ \mathrm{cm}^{3}$. The SERS spectrum is taken with volume of BPE solution $=0.5 \mathrm{ml}$, and the area of the nanopillars substrate sample is about $0.5 \mathrm{~cm}^{2}$. Thus, for a concentration of $35 \mathrm{pM}$, a upper bound for the BPE areal density on the substrate is $N_{\text {SERS }}=2.1 \times 10^{10}$ molecules $/ \mathrm{cm}^{2}$. Note that the BPE areal density in a monolayer can be assumed to be $7 \times 10^{14}$ [17], so the coverage here is $<0.001$ monolayer. For our system, $L$ is estimated to be $0.02 \mathrm{~cm}$. Therefore, by substituting these numbers into eq. (1), we estimate the enhancement to be $E F=5.8 \times 10^{8}$.

It is previously shown that this type of straight nanopillar substrate can support resonances arising from cavity modes of coupled surface plasmons in the gap between the pillars [9]. By numerical simulation, resonance of order 3 was previously observed in this structure at a wavelength of $650 \mathrm{~nm}$, which is close to the excitation wavelength of $660 \mathrm{~nm}$ used in the SERS experiment here. The cavity effect leads to 3 anti-nodes inside the pillar gap, which can serve as "hot spots" for SERS enhancement. From the simulation, the local electric field at the cavity anti-nodes can be enhanced by as much as 2 orders-of-magnitude, which leads to the electromagnetic enhancement of the SERS signal for molecules adsorbed on the metal within the hotspots. Current work includes extending the application of the VNPs to gas analysis. 


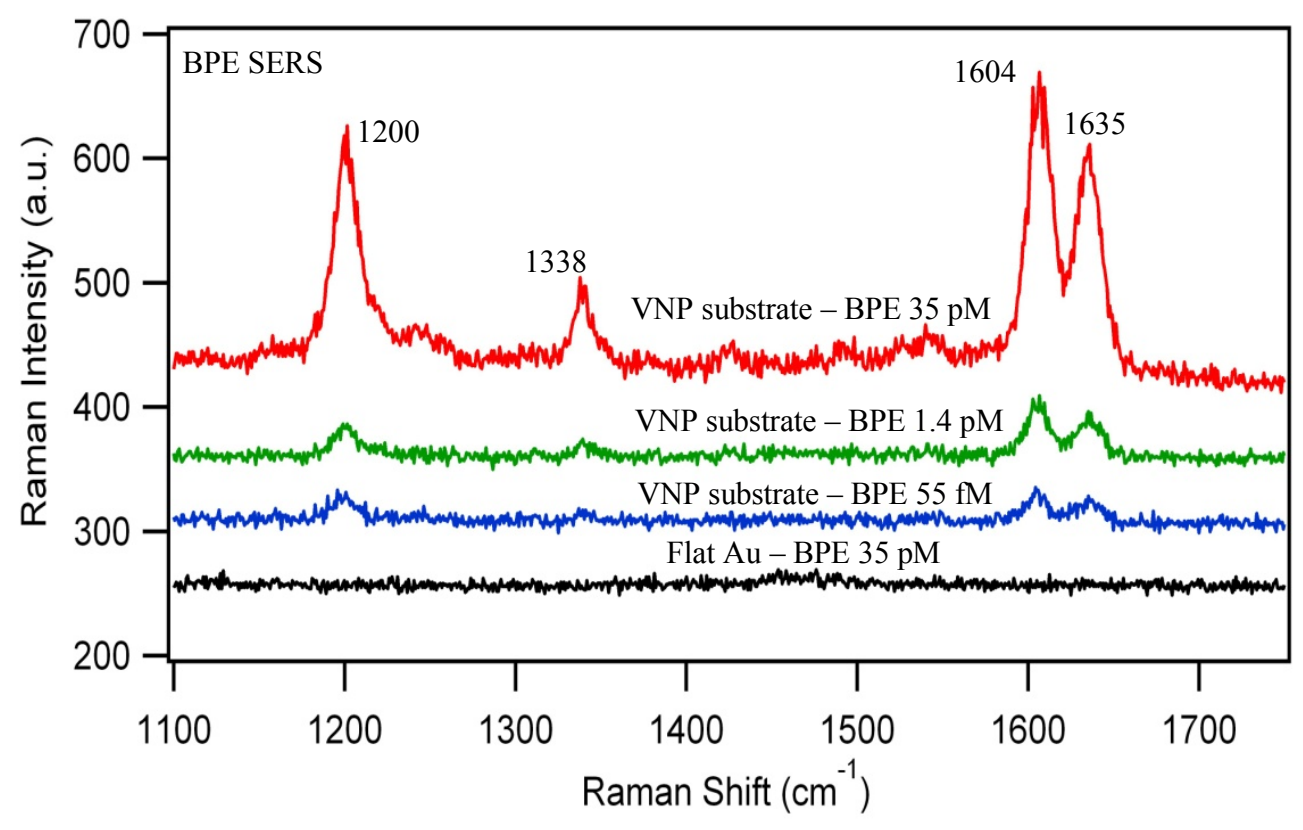

a.)

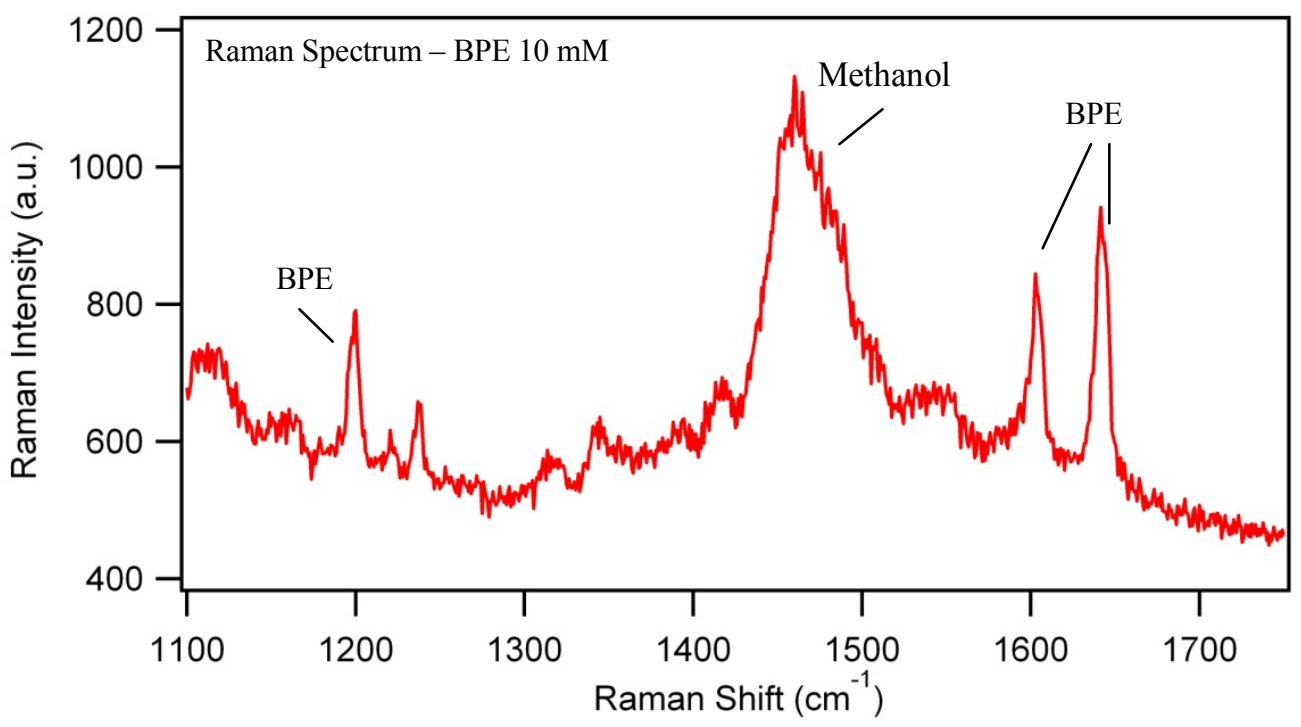

b.)

Fig. 2. a.) SERS spectra of BPE at concentrations of $35 \mathrm{pM}, 1.4 \mathrm{pM}$ and $55 \mathrm{fM}$ using vertical nanopillar (VNP) array, showing that concentration down to femto-molar level can be detected; b.) Raman spectrum of $10 \mathrm{mM}$ BPE obtained using the same spectroscopy system. Data acquisition time is 10 secs for (a.) and $300 \mathrm{secs}$ for (b.). 


\subsection{SERS of toluene vapor using Ag-coated tapered nanopillar (TNP) substrate}

The Ag-coated TNP substrates have previously shown enhancement factor of around $10^{8}$ with benzenethiol (BT) monolayer [14]. As toluene has a similar molecular structure as BT, we continued on to use the TNP substrate for detecting toluene vapor. It is also previously reported that out of several metal coatings, Ag provides the largest enhancement for toluene vapor and a number of other organic molecules [3], suggesting that it is more favorable to binding with the molecules.

The SERS spectrum of toluene vapor (mixed with $\mathrm{N}_{2}$ as the carrier gas) obtained at substrate temperature of $25{ }^{0} \mathrm{C}$ using the TNP substrate depicted in Fig $1 \mathrm{~d}$. are shown in fig. 3a. The concentration of the toluene vapor in the gas mixture is estimated to be around 3\% (by partial pressure) assuming that the toluene vapor saturates the $\mathrm{N}_{2}$ carrier gas in the bubbling process. The spectrum was taken after the toluene/ $\mathrm{N}_{2}$ gas mixture flows for 15 mins. The laser wavelength is $532 \mathrm{~nm}$ and the measured laser power is $4 \mathrm{~mW}$. The data acquisition time is 60 secs. Also shown in fig. $3 \mathrm{a}$ is the TNP substrate background taken in pure $\mathrm{N}_{2}$ ambient. The background shows a broad band around $1500-1600 \mathrm{~cm}^{-1}$, which is most likely due to carbon contamination on the substrate surface. For comparison, the Raman spectrum of neat toluene liquid obtained using the same laser power and data acquisition time is shown in fig. $3 \mathrm{~b}$. Raman spectroscopy was attempted on the toluene vapor/ $\mathrm{N}_{2}$ gas mixture using the same experimental conditions but no discernible toluene peak was observed after up to 30 mins. of data acquisition time.

Fig. 3 shows that while many of the characteristic Raman peaks of toluene are recognizable in the SERS spectrum, it is somewhat different from the Raman spectrum. The characteristic Raman peaks of toluene at 1002, 1031, 1605 (due to $\mathrm{C}=\mathrm{C}$ stretching) were shifted slightly in the SERS spectrum to 994,1024 , and 1594 , respectively. The 1210 peak in the bulk Raman spectrum (due to ring-CH3 stretching) maintained its position in the SERS spectrum, but is broadened. Interestingly, the 522 (ring deformation) and 789 (ring-CH3 stretching) peaks in the bulk Raman spectrum were suppressed in the SERS spectrum (and may be shifted), while a new, broad band at 637 appears. This band is very repeatable, and it is not previously reported. It is not clear at this point if it is due to the shifting of the 622 Raman peak (ring deformation). These features strongly suggest that the SERS spectrum is not due to the Raman scattering of liquid toluene condensed onto the TNP substrate, but is indeed due to adsorbed toluene molecules that interact with the Ag surface to shift original peaks as well as produce new peaks.

Furthermore, the kinetics and temperature dependence of the SERS signal were also investigated. The results show that the toluene vapor SERS signal increases monotonically with time and saturates after around 12 mins. at substrate temperature of $25{ }^{\circ} \mathrm{C}$. The SERS signal also increases with decreasing substrate temperature. At $0{ }^{\circ} \mathrm{C}$, which is well below the condensation temperature, there is visibly condensation of the toluene onto the substrate after around 10 mins. The 3 strongest peaks in the SERS spectrum are still apparent and persistent even after the toluene vapor flow is stopped and the sample kept in pure $\mathrm{N}_{2}$ for 3 days. Nevertheless, they can be completely purged out by heating the sample at around $110{ }^{\circ} \mathrm{C}$ for 5 mins. These features suggest that the SERS signal can be dynamically controlled by thermal adsorption and desorption cycle.

It is known that a sharp metallic tip can confine electromagnetic field tightly at its tip due to the lightening rod effect, leading to large localized field enhancement [19]. The metallic coating on the TNPs provides the support for excitation of surface plasmons resonances for the lightening rod effect. The TNP thus serves as a nano-focusing device that adiabatically focus the light energy onto the tip of the pillars. This nano-focusing effect thus creates the SERS "hot spots" [20] necessary for the electromagnetic field enhancement. Simulation results by density functional theory have previously shown that the interaction between a binding organic molecule and metal surface can lead to a shift in Raman peaks and contribute additional enhancement to the SERS signal due to presence of a strong electrostatic built-in field [21]. This interaction can thus lead to drastic changes in SERS spectrum as compared to Raman spectrum. Current work includes extending the application of the TNPs to other gases.

\section{CONCLUSIONS}

SERS was demonstrated for a new class of substrates: the vertical nanopillars (VNP) and tapered nanopillars (TNP) arrays, using BPE and toluene vapor, respectively. BPE can be detected down to fM concentration level with Au-coated VNP substrates that show enhancement factor of over $0.5 \times 10^{9}$. Toluene vapor can be detected by the Ag-coated TNP 
substrates at room temperature. The observed toluene vapor SERS spectrum shows slightly shifted Raman peaks as well as new strong SERS band at $637 \mathrm{~cm}^{-1}$. The results show the promising potential for these types of substrates in robust, high-sensitivity environmental sensing using SERS for detecting chemicals and gases.

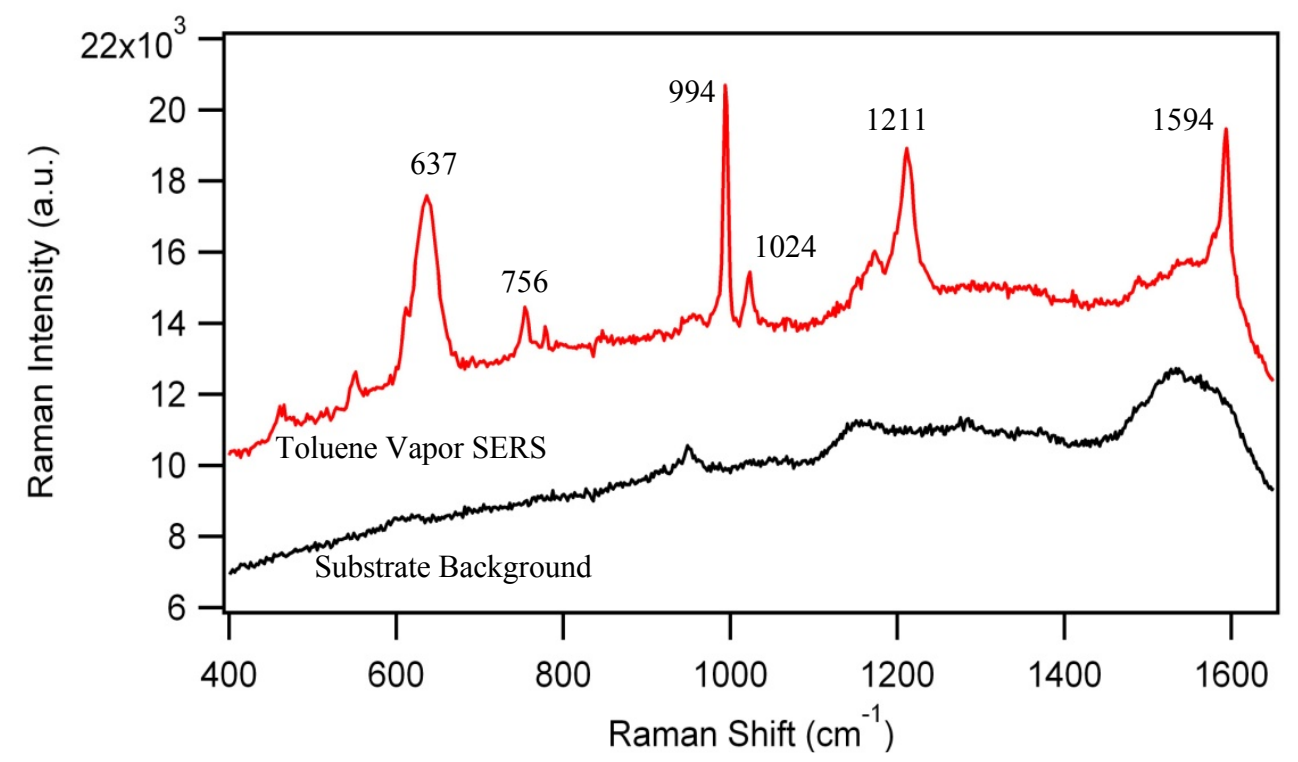

a.)

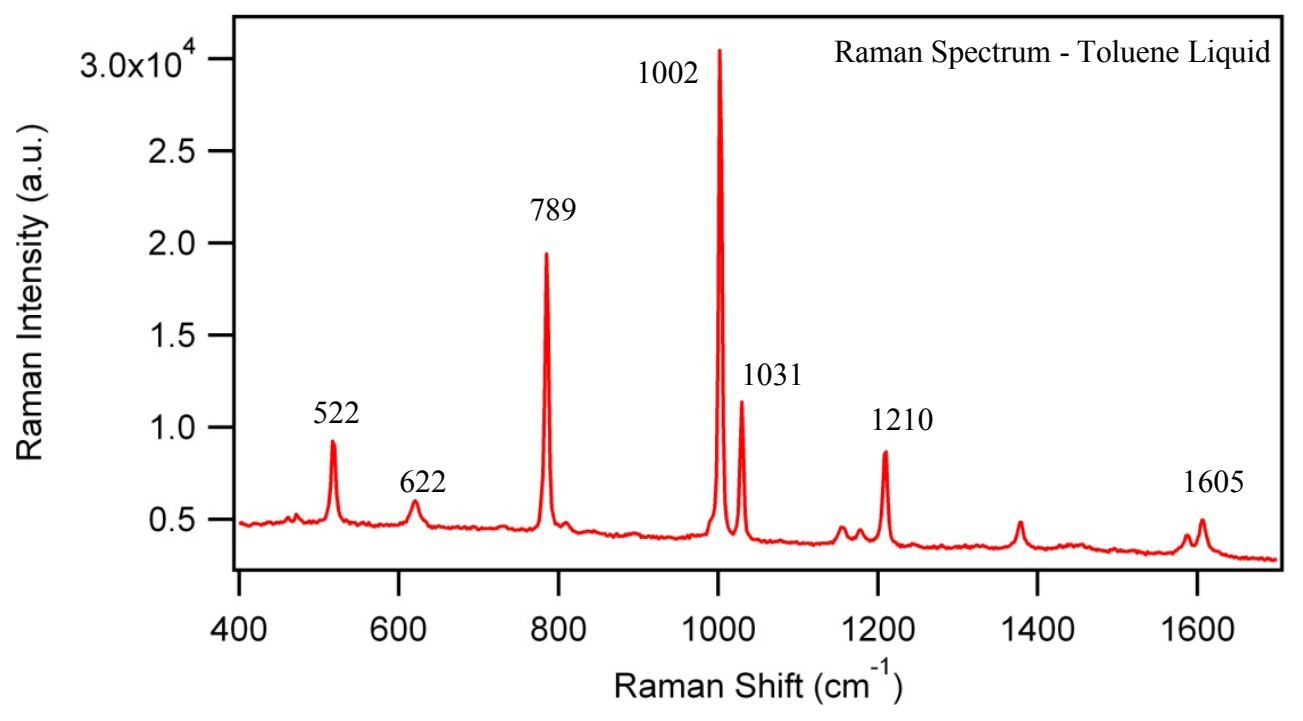

b.)

Fig. 3. a.) SERS spectrum of toluene vapor using tapered nanopillar (TNP) array at substrate temperature of $25{ }^{\circ} \mathrm{C}$, the toluene vapor is carried by nitrogen and has an estimated concentration of around $3 \%$ by partial pressure; b.) Raman spectrum of neat toluene liquid obtained using the same spectroscopy system. 


\section{REFERENCES}

[1] Kneipp, K., Wang, Y., Kneipp, H., Perelman, L.T., Itzkan, I., Dasari, R.R., and Feld, M.S., "Single molecule detection using surface-enhanced Raman scattering (SERS)," Phys. Rev. Lett. 78(9), 1667-1670 (1997).

[2] Nie, S. and Emory, S.R., "Probing single molecules and single nanoparticles by surface-enhanced Raman scattering," Science 275(5303), 1102-1106 (1997).

[3] Wachter, E.A., Storey, J.M.E., Sharp, S.L., Carron, K.T., and Jiang, Y., "Hybrid substrates for real-time SERSbased chemical sensors,"Appl. Spectrosc. 49(2), 193-199 (1995).

[4] Mosier-Boss, P.A., and Lieberman, S.H., "Detection of volatile organic compounds using surface enhanced Raman spectroscopy substrates mounted on a thermoelectric cooler," Analytica Chimica Acta 488(1), 15-23 (2003).

[5] Biggs, K.B., Camden, J.P., Anker, J.N., and Van Duyne, R.P., "Surface-enhanced Raman spectroscopy of benzenethiol adsorbed from the gas phase onto silver film over nanosphere surfaces: determination of the sticking probability and detection time limit," J. Phys. Chem. A 113(16), 4581-4586 (2009).

[6] Rae, S.I., and Khan, I., "Surface enhanced Raman spectroscopy (SERS) sensors for gas analysis," Analyst 135, 1365-1369 (2010).

[7] Zeisel, D., Deckert, V., Zenobi, R., and Vo-Dinh, T., "Near-field surface-enhanced Raman spectroscopy of dye molecules adsorbed on silver island films," Chem. Phys. Lett. 283(5-6), 381-385 (1998).

[8] Murray, C.A., Allara, D.L., and Rhinewine, M., "Silver-molecule separation dependence of surface-enhanced Raman scattering," Phys. Rev. Lett. 46(1), 57-60 (1981).

[9] Talley, C.E., Jackson, J.B., Oubre, C., Grady, N.K., Hollars, C.W., Lane, S.M., Huser, T.R., Nordlander, P., and Halas, N.J., "Surface-enhanced Raman scattering from individual $\mathrm{Au}$ nanoparticles and nanoparticle dimer substrates," Nano Lett. 5(8), 1569-1574 (2005).

[10] Haynes, C.L., and Van Duyne, R.P., "Nanosphere lithography: a versatile nanofabrication tool for studies of sizedependent nanoparticle optics," J. Phys. Chem. B 105(24), 5599-5611 (2001).

[11]Im, H., Bantz, K.C., Lindquist, N.C., Haynes, C.L., and Oh, S.H., "Vertically oriented sub-10-nm plasmonic nanogap arrays," Nano Lett. 10(6), 2231-2236 (2010).

[12] Li, W.D., Ding, F., Hu, J., and Chou, S.Y., "Three-dimensional cavity nanoantenna coupled plasmonic nanodots for ultrahigh and uniform surface-enhanced Raman scattering over large area," Opt. Express 19(5), 3925-3936 (2011).

[13] Bora, M., Fasenfest, B.J., Behymer, E.M., Chang, A.S.P., Nguyen, H.T., Britten, J.A., Larson, C.C., Chan, J.W., Miles, R.R., and Bond, T.C., "Plasmon resonant cavities in vertical nanowire arrays," Nano Lett. 10(8), 2832-2837 (2010).

[14] Gartia, M.R., Xu, Z., Behymer, E., Nguyen, H., Britten, J.A., Larson, C., Miles, R., Bora, M., Chang, A.S.P., Bond, T.C., and Liu, G.L., "Rigorous surface enhanced Raman spectral characterization of large-area high-uniformity silver-coated tapered silica nanopillar arrays," Nanotechnology 21, 395701 (2010).

[15]Fernandez, A., Nguyen, H.T., Britten, J.A., Boyd, R.D., Perry, M.D., Kania, D.R., and Hawryluk, A.M., "Use of interference lithography to pattern arrays of submicron resist structures for field emission flat panel displays," J. Vac. Sci. Technol. B 15(3), 729-735 (1997).

[16] Mayer, B., Collins, C.C., and Walton, M., "Transient analysis of carrier gas saturation in liquid source vapor generators," J. Vac. Sci. Technol. A 19(1), 329-344 (2001).

[17] Norrod, K.L., Sudnik, L.M., Rousell, D., and Rowlen, K.L., "Quantitative comparison of five SERS substrates: sensitivity and limit of detetction," Appl. Spectrosc. 51(7), 994-1001 (1997).

[18] Aggarwal, R.L., Farrar, L.W., Diebold, E.D., and Polla, D.L., "Measurement of the absolute Raman scattering cross section of the 1584-cm-1 band of benzenethiol and the surface-enhanced Raman scattering cross section enhancement factor for femtosecond laser-nanostructured substrates," J. Raman Spectrosc. 40(9), 1331-1333 (2009).

[19] Stockman, M.I., "Nanofocusing of optical energy in tapered plasmonic waveguides," Phys. Rev. Lett. 93, 137404 (2004).

[20] Wu, W., Hu, M., Ou, F.S., Li, Z., and Williams, R.S., “Cones fabricated by 3D nanoimprint lithography for highly sensitive surface enhanced Raman spectroscopy," Nanotechnology 21, 255502 (2010).

[21] Gartia, M.R., Bond, T.C., and G. L. Liu, "Metal-molecule Schottky junction effects in surface enhanced Raman scattering,” J. Phys. Chem. A 115(3), 318-328 (2011).

\section{ACKNOWLEDGEMENT}

This work was performed under the auspices of the U.S. Department of Energy by Lawrence Livermore National Laboratory under contract DE-AC52-07NA2734. 